\title{
EYE-FIXATION RELATED POTENTIAL TO STIMULI SIMULATING THE VISION OF AN OLDER ADULT
}

\author{
Koji KAZAI'), Yuka ABE'), Akihiro YAGI') \\ ${ }^{1)}$ Kwansei Gakuin University, Japan \\ Tomoaki NAKANO ${ }^{2)}$, Kazunori HIGUCHI ${ }^{2)}$ and Shin YAMAMOTO ${ }^{2)}$ \\ ${ }^{2)}$ Toyota Central R\&D Laboratories, Inc., Japan
}

\begin{abstract}
The eye-fixation related potential (EFRP) is a type of visual evoked potential (VEP). In the present study, the effects of simulating the vision of an older adult (Nakano, Higuchi, \& Yamamoto, 1996) on the EFRP of young adults were investigated. The EFRP was measured under three conditions: the $\mathrm{N}$ condition (original stimulus without any image processing), the CS condition (simulation of a 70-year old person's contrast sensitivity and spectral transmittance), and the $\mathrm{C}$ condition (simulation of a 70-year old person's contrast sensitivity). The P65 (positivity with a peak latency of about $65 \mathrm{~ms}$ ) and N110 (nagativity with a peak latency of about $110 \mathrm{~ms}$ ) of the EFRP were identified. There was no significant difference between the P65-N110 amplitude of the N condition and that of the CS condition. The peak latency of P65 of the CS condition was longer than those of the other two conditions. These results are in general agreement with previous VEP studies that compared older and young adults. Therefore, these results support the validity of the simulation.
\end{abstract}

Key words: eye-fixation related potential (EFRP), aging, simulation, contrast sensitivity

Visual evoked potential (VEP), or visual event-related potential (ERP), is used as a tool for examining visual cognition (e.g., Desmedt, 1977; Regan, 1989). The eye-fixation related potential (EFRP) is a type of VEP that is used for examining visual cognition with eye movements. The EFRP is obtained by averaging occipital EEGs time-locked to the offset of saccadic eye movements (saccades). The EFRP is nearly synonymous with the lambda complex (e.g., Marton \& Szirtes, 1982; Yagi, 1979) or the lambda wave (Kurtzberg \& Vaughan, 1973; Thickbroom, Knezevic, Carroll, \& Mastaglia, 1991). The most prominent component of the EFRP is the lambda response, which is a sharp positive component with a latency of about $70 \mathrm{~ms}$ from the offset of saccades. The lambda response is usually followed by a negativity peak at around $110 \mathrm{~ms}$. The lambda response

This research was supported by a Grant-in Aid for Scientific Research (11551003), a Grant in Academic Frontier Promotion Project provided by MEXT.

Corresponding concerning this article should be sent to Koji Kazai, Department of Psychology, Kwansei Gakuin University, Uegahara 1-1-155, Nishinomiya 662-8501, Japan (e-mail: kazai@kwansei.ac.jp).

${ }^{1)}$ Department of Psychology, Kwansei Gakuin University, Japan.

${ }^{2)}$ Human Factors Lab., Toyota Central R\&D Labs., Inc., Japan.

Current affiliation of Tomoaki Nakano and Shin Yamamoto: Department of Information Engineering, Faculty of Science and Technology, Meijo University, Japan. 
had been considered to occur at the onset of saccades (Kurtzberg \& Vaughan, 1973). Later studies, however, have indicated that the lambda response and some components are associated with the offset of saccades (i.e., the onset of eye fixation) (Billings, 1989; Marton \& Szirtes, 1982; Thickbroom et al., 1991; Yagi, 1979). This finding suggests that these components of the EFRP reflect visual information processing after the offset of saccades. Indeed, some studies have shown that the lambda response is affected by the physical properties of the stimulus (e.g., Kazai \& Yagi, 1999; Marton \& Szirtes, 1982) and by psychological factors (Yagi, 1981). The EFRP has the advantage of allowing participants to move their eyes, while the recording of so-called VEPs requires the restriction of a participant's eye movements.

It is well known that visual functions decline during normal aging. This means that older and younger adults perceive objects differently. Therefore, ergonomic designs should vary according to the age of the target population, and designs for older adults should consider specifications suitable for their particular group. For this purpose, Nakano, Higuchi, and Yamamoto (1996) developed a system that can simulate the visual functions of an older adult on a computer monitor. This system can simulate the decline in three properties of vision: contrast sensitivity, spectral transmittance of crystalline lens, and accommodation. These properties were calculated from psychophysical data, and the simulation was not evaluated by electrophysiological measurements. In the present study, the EFRP was used to evaluate the simulation as an electrophysiological measurement.

\section{METHODS}

Subjects:

Eleven students (4 male and 7 female), 20-22 years of age, participated in the experiment. They had normal or corrected-to-normal visual acuity. Informed consent was obtained after the experimental procedures were explained.

\section{Stimulus and Apparatus:}

The stimuli were presented on a 17-inch CRT display (Nanao Flexscan E55D), which was placed at a distance of $60 \mathrm{~cm}$ from the eyes of the subjects. Each subject was seated in front of the CRT display in a soundproof, shielded room. A head rest was used to maintain the position of the head.

Fig. 1 shows an example of the stimuli. The stimuli consisted of $\mathrm{Cs}\left(0.3^{\circ}\right.$ in diameter $)$ and bars that were drawn on a black background. The Cs were arranged in a $6 \times 6$ array, and the bars were located between the Cs. The distance between Cs was $4^{\circ}$. The Cs were rotated $0^{\circ}, 45^{\circ}, 90^{\circ}, 135^{\circ}, 180^{\circ}, 225^{\circ}, 270^{\circ}$, or $315^{\circ}$.

There were three conditions according to the image processing: $\mathrm{N}, \mathrm{CS}$, and $\mathrm{C}$. The stimulus of the $\mathrm{N}$ condition was an original stimulus of white items on a black background without any image processing (maximum luminance: $89 \mathrm{~cd} / \mathrm{m}^{2}$ ). The CS condition was a simulation of a 70 -year-old person's contrast sensitivity and spectral transmittance (maximum luminance: $50 \mathrm{~cd} / \mathrm{m}^{2}$ ). The $\mathrm{C}$ condition was a simulation of a 70 -year-old person's contrast sensitivity (maximum luminance: $89 \mathrm{~cd} / \mathrm{m}^{2}$ ). Their luminance contrasts were more than $96 \%$.

Although the CS condition did not simulate the accommodation property, it was used to replicate the vision of an older adult because the distance from subject's eyes to the display was kept constant.

\section{Procedure:}

The stimulus was presented for 25 seconds as one trial. The subjects were instructed to search for Cs that were rotated in a specific direction. Each stimulus contained 5 to 12 target Cs. The subjects performed 7 


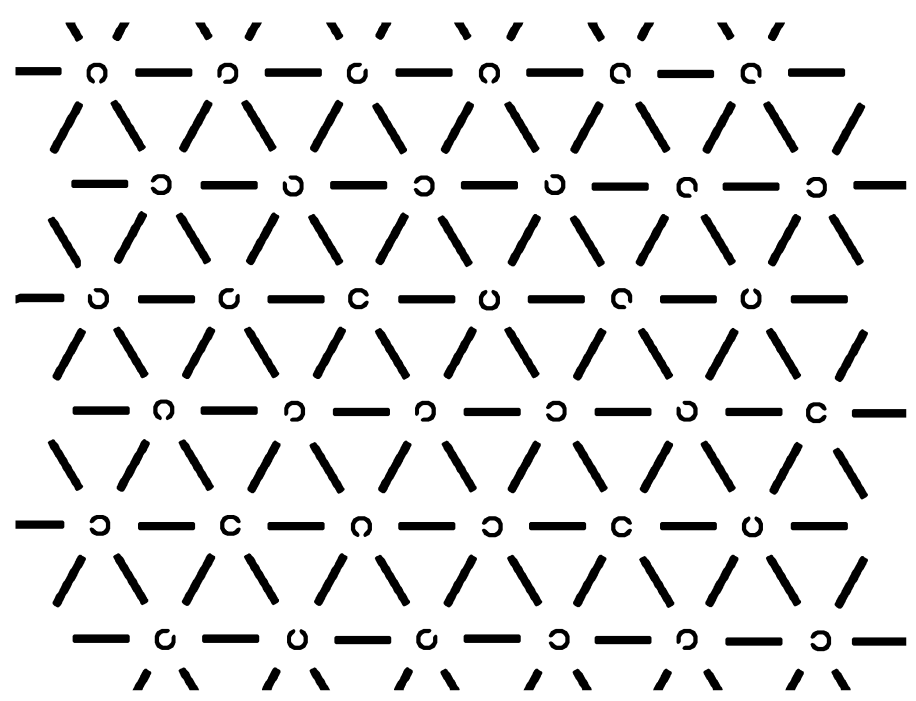

Fig. 1. An example of the stimuli. The subjects were instructed to search Cs that rotated in a specific direction. There were three conditions according to the image processing. The $\mathrm{N}$ condition consisted of an original stimulus of white items on a black background without any image processing. The CS condition consisted of a simulation of a 70-year-old person's contrast sensitivity and spectral transmittance. The $\mathrm{C}$ condition consisted of a simulation of a 70-year-old person's contrast sensitivity.

trials for each condition. The direction of the target Cs was delivered to the subject before the trial. The stimuli were presented in randomized order across the conditions.

\section{Recording:}

EEGs were recorded from four occipital sites, $\mathrm{O} 1, \mathrm{O} 2, \mathrm{Oz}$, and POz. Linked earlobes were used as the reference. The ground lead was attached to the midline forehead. Eye movements were recorded by means of EOGs. A pair of $\mathrm{Ag}-\mathrm{AgCl}$ electrodes was placed at the outer canthi of the two eyes for horizontal eye movements. Another pair of electrodes was placed above and below the left eye for vertical eye movements and eye-blinking potentials.

The EEGs and EOGs were amplified with differential amplifiers (Nihon Kohden AB-621G) at a time constant of $2.0 \mathrm{~s}$ and a high frequency cut off of $100 \mathrm{~Hz}$. All of these signals were digitized every $2 \mathrm{~ms}$ with a 14-bit A/D converter (TEAC PS9354).

Data Analysis:

All raw EEGs and EOGs were reviewed off-line on a computer terminal. The offset points of the saccades were determined by moving a graphic cursor manually on the horizontal EOG tracing on the computer terminal. The EEGs without artifacts between $200 \mathrm{~ms}$ before and $300 \mathrm{~ms}$ after the offset point of the saccade were averaged. More than 48 epochs were averaged for each condition. The directions of saccades were counterbalanced.

Repeated measures analyses of variance (ANOVAs) were conducted on data with Greenhouse-Geisser epsilon correction, but the original degrees of freedom were reported to aid the interpretation of the statistical designs. The Newman-Keuls test was used for post-hoc comparisons. An alpha level of .05 was used for all statistical tests. 
01
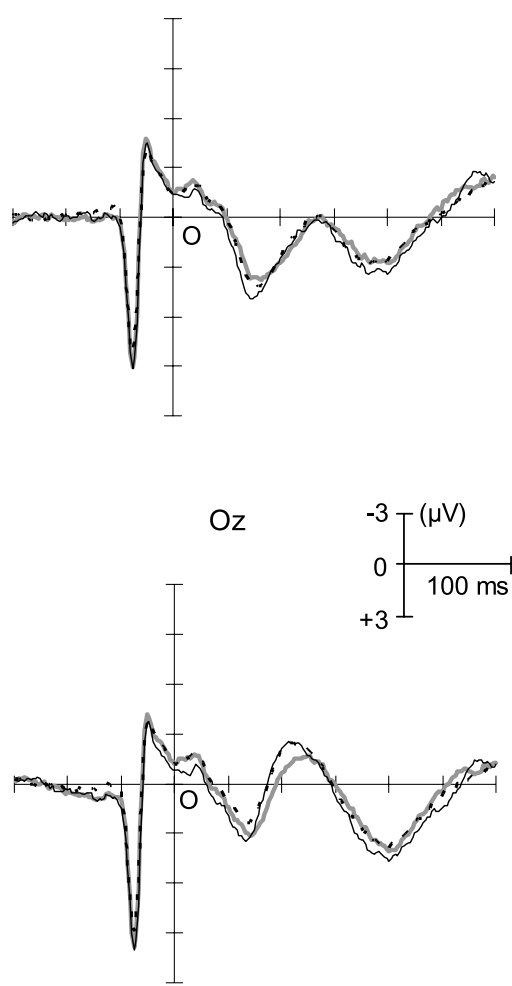

$\mathrm{O} 2$
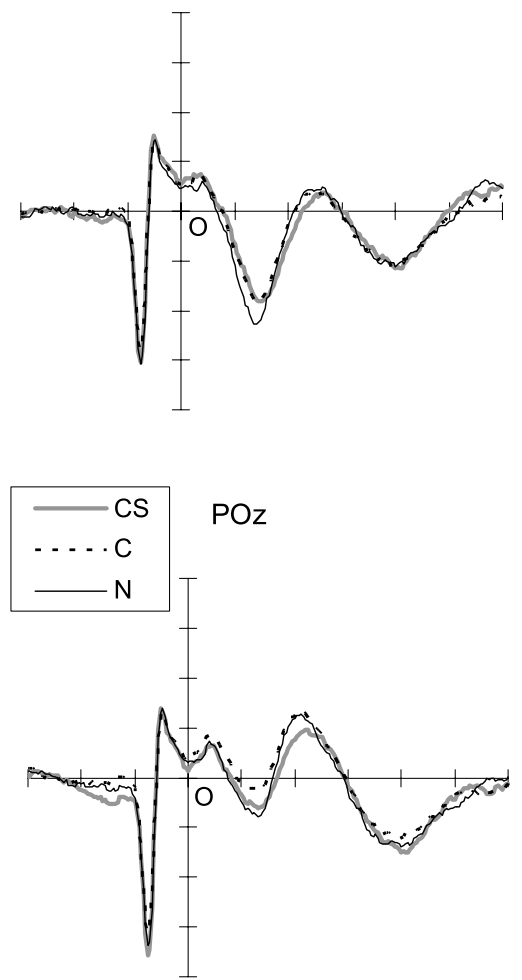

Fig. 2. Grand-averaged waves of EFRP overplotted for all the conditions. The origin indicates the offset of a saccade.

\section{RESULTS}

Fig. 2 shows the grand-averaged waves of the EFRP over 11 subjects under the three conditions. The baseline was computed as the mean of all data points from 75 to 51 prior to the offset point of the saccade. Positive components (lambda response: P65) and negative components (N110) appeared in all conditions, with a peak latency of approximately $65 \mathrm{~ms}$ and $110 \mathrm{~ms}$, respectively, from the offset point of the saccades. The $\mathrm{P} 65$ and N110 components observed in Oz were used for subsequent data analysis because previous VEP studies (Emmerson-Hanover, Shearer, \& Creel, 1994; Page \& Crognale, 2005) have also analyzed the waves at Oz. The peak amplitude was defined as the P65N110 peak to peak amplitude.

A repeated measures ANOVA revealed a significant effect of condition on peak amplitude $(F(2,20)=5.34, p<.05)$. Further analysis with the Newman-Keuls test indicated that the peak amplitude of the $\mathrm{C}$ condition (mean $=5.9 \mu \mathrm{V}, S D=2.73$ ) was significantly smaller than the peak amplitude of the $\mathrm{N}$ condition (mean $=7.0 \mu \mathrm{V}, S D=3.04$ ) and that the peak amplitude of the $\mathrm{C}$ condition was marginally smaller than that of the CS condition 
(mean $=6.5 \mu \mathrm{V}, S D=3.1)(p=.08)$.

A repeated measures ANOVA revealed a significant effect of condition on P65 latency $(F(2,20)=4.82, p<.01)$. Further analysis by the Newman-Keuls test indicated that the latency of the $\mathrm{CS}$ condition (mean $=66 \mathrm{~ms}, S D=13.6$ ) was significantly longer than the latency of the $\mathrm{C}$ condition (mean=60 ms, $S D=15.7$ ) and that the latency of the $\mathrm{CS}$ condition was marginally longer than that of the $\mathrm{N}$ condition (mean=62 ms, $S D=12.0$ ) $(p=.07)$. There was no significant effect of condition on the N110 latency $(F=1.16)$.

The mean percentage of the correct trials was $92.2 \%$ for the $\mathrm{N}$ condition, $89.6 \%$ for the CS condition, and $88.3 \%$ for the $\mathrm{C}$ condition. There were no significant differences in accuracy between the conditions.

\section{DISCUSSION}

The present study investigated the effects of the simulation of the visual functions of an older adult (Nakano et al., 1996) on the EFRP. The main results of the present study relate to changes in the P65-N110 amplitude and the increase of the P65 latency, which were caused by the simulation of the visual functions of an older adult.

The P65-N110 amplitude of the C condition (simulation of the contrast sensitivity of an older adult) was smaller than those of the $\mathrm{N}$ condition (original stimulus) and the CS condition (simulation of both the contrast sensitivity and spectral transmittance of an older adult), and there was no significant difference between the $\mathrm{N}$ and CS conditions. These results suggest that the spatial frequency and the spectral components affect the P65-N110 amplitude differently.

The difference in amplitude between the $\mathrm{N}$ and $\mathrm{C}$ conditions can be attributed to the difference in the spatial frequency components they have because other stimulus properties (e.g., the luminance level) are the same between them. The spatial frequency components of the $\mathrm{N}$ condition are different from those of the $\mathrm{C}$ condition since higher spatial frequency components are reduced in the $\mathrm{C}$ condition. Thus, the present result indicates that the P65-N110 amplitude is affected by the spatial frequency components of the stimulus. This result is consistent with previous studies (Kazai \& Yagi, 1999; Scott, Groethuysen, \& Bickford, 1967). For example, Kazai and Yagi showed a linear correlation between the P65-N110 amplitudes and the logarithm of check sizes: the P65N110 amplitude decreased as the fundamental spatial frequency of the stimulus decreased.

It is interesting that the amplitude of the CS condition is larger than that of the $\mathrm{C}$ condition in that the lower luminance level stimulus elicited a larger potential. This result might appear inconsistent with a general finding that a higher luminance level stimulus elicits a larger VEP (e.g., Brannan, Solan, Ficarra, \& Ong, 1998; Tobimatsu, KuritaTashima, Nakayama-Hiromatsu, Akazawa, \& Kato, 1993). This discrepancy is probably due to the simulation of spectral transmittance under the CS condition. The simulation of the spectral transmittance of an older adult might have compensated for the amplitude decrease caused by the simulation of the spatial frequency components. Thus, the closer simulation of an older adult had no effect on the P65-N110 amplitude, while the partial 
simulation of an older adult decreased the amplitude. In fact, VEP studies that compared older adults with young adults reported no significant difference in the VEP amplitude between them (Tobimatsu et al., 1993; Page \& Crognale, 2005).

As for the P65 latency, the CS condition was longer than those of the other two conditions. This result is probably due to the lower luminance level under the CS condition than under the other two conditions. This idea is supported by previous studies that showed that the peak latency of the lambda response increased as the luminance level decreased (Gaarder, Krauskopf, Graf, Kropfl, \& Armington, 1964; Scott et al., 1967). Furthermore, there was no significant difference between the $\mathrm{N}$ and $\mathrm{C}$ conditions. This result implies that the P65 latency was unaffected by the difference in the spatial frequency components.

The effect of the simulation on the P65 latency might disagree with a recent VEP study that compared young adults with older adults (Page \& Crognale, 2005). Page and Crognale reported that the latency increased with age when a chromatic stimulus was used but not when an achromatic (black and white) stimulus was used. It is probable that this disagreement arises from the difference in the stimulus properties. Page and Crognale used a stimulus of a single spatial frequency component (sinusoidal grating of $1 \mathrm{cpd}$ ) and low contrast (eight times greater than the contrast threshold). In contrast to the experiment by Page and Crognale, however, the VEP studies that used an achromatic (black and white) and more complex stimulus (checkerboard pattern) of higher contrast (more than $80 \%$ ) reported that there was a significant effect of aging on the VEP peak latency (Emmerson-Hanover, Shearer, \& Creel, 1994; Tobimatsu et al., 1993).

In conclusion, the fact that previous VEP studies and the present study are in general agreement demonstrates the validity of the system that simulates the vision of older adults (Nakano et al., 1996). Future research should investigate the effects of the simulation of young adults on the behavior and electrophysiological responses of older adults.

\section{REFERENCES}

Billings, R. J. 1989. The origin of the occipital lambda wave in man. Electroencephalography and Clinical Neurophysiology, 72, 95-113.

Brannan, J. R., Solan, H. A., Ficarra, A. P., \& Ong, E. 1998. Effect of luminance on visual evoked potential amplitudes in normal and disabled readers. Optometry and Vision Science, 75, 279-283.

Desmedt, J. E. 1977. Visual evoked potentials in man, new developments. New York: Clarendon.

Emmerson-Hanover, R., Shearer, D. E., \& Creel, D. J. 1994. Pattern reversal evoked potentials: gender differences and age-related changes in amplitude and latency. Electroencephalography and Clinical Neurophysiology, 92, 93-101.

Gaarder, K., Krauskopf, J., Graf, V., Kropfl, W., \& Armington, J. C. 1964. Averaged brain activity following saccadic eye movement. Science, 146, 1481-1483.

Kazai, K., \& Yagi, A. 1999. Integrated effect of stimulation at fixation points on EFRP (eye-fixation related brain potentials). International Journal of Psychophysiology, 32, 193-203.

Kurtzberg, D., \& Vaughan, H. G. Jr. 1973. Electrocortical potentials associated with eye movements. In V. Zikmund (Ed.), The oculomotor system and brain functions (pp. 137-146). London: Butterworths.

Marton, M., \& Szirtes, J. 1982. Averaged lambda potential and visual information processing. Studia Psychologica, 24, 165-170. 
Nakano, T., Higuchi, K., \& Yamamoto, S. 1996. Visualization system for experiencing visual functions of elderly people by imaging processing. The Journal of the Institute of Television Engineers of Japan, 50, 1489-1495. (In Japanese with English Abstract)

Page, J. W., \& Crognale, M. A. 2005. Differential aging of chromatic and achromatic visual pathways: behavior and electrophysiology. Vision Research, 45, 1481-1489.

Regan, D. 1989. Human brain electrophysiology: evoked potentials and evoked magnetic fields in science and medicine. New York: Elsevier.

Scott, D. F., Groethuysen, U. C., \& Bickford, R. G. 1967. Lambda responses in the human electroencephalogram. Neurology, 17, 770-778.

Thickbroom, G. W., Knezevic, W., Carroll, W. M., \& Mastaglia, F. L. 1991. Saccade onset and offset lambda waves: relation to pattern movement visually evoked potentials. Brain Research, 551, 150156.

Tobimatsu, S., Kurita-Tashima, S., Nakayama-Hiromatsu, M., Akazawa, K., \& Kato, M. 1993. Age-related changes in pattern visual evoked potentials: differential effects of luminance, contrast and check size. Electroencephalography and Clinical Neurophysiology, 88, 12-19.

Yagi, A. 1979. Saccade size and lambda complex in man. Physiological Psychology, 7, 370-376.

Yagi, A. 1981. Visual signal detection and lambda responses. Electroencephalography and Clinical Neurophysiology, 52, 604-610.

(Manuscript received March 30, 2005; Revision accepted May 18, 2005) 